\title{
Dissecting RAS Oncogene-Induced Kinome Involved in Breast Cancer Metastasis Amna Yaqub ${ }^{1}$, Harikrishna Nakshatri ${ }^{2}$
}

${ }^{1}$ Indiana University School of Medicine; ${ }^{2}$ Indiana University School of Medicine, Department of Surgery

\section{Background and Hypothesis:}

The RAS and PI3K-AKT-mTOR signaling pathways are often dysregulated in cancer. RAS pathway alterations, however, are more common in breast cancer metastasis. The laboratory's recently developed model system demonstrated the ability of RAS but not PIK3CA-induced signals in promoting metastasis of breast cancer. Unbiased kinome analyses of isogenic RAStransformed primary tumor and metastatic cells and PIK3CA-transformed primary tumor cells enabled identification of RAS-activated kinome, which included FER, PAK4, LIMK1, PIK3CD and Casein Kinase 2 (CK2). We hypothesized that therapeutic targeting of these kinases may reduce breast cancer metastasis. As a proof-of-principle, the effect of the CK2 inhibitor Silmitasertib, which is in clinical trial for COVID-19 and refractory multiple myeloma, was tested.

\section{Experimental Design:}

The study included four isogenic cell lines: "normal" (KTB34-hTERT), PIK3CA-transformed (TKTB34-PIK3CA), RAS-transformed (TKTB34-RAS), and RAS-transformed cells metastasized to lungs (MKTB34-RAS). Active kinomes in these cells were identified using phosphoproteomics and functional kinome profiling using multiplexed kinase inhibitor beads. Expression levels of FER, PAK4, LIMK1, and PIK3CD kinases were compared through Western Blot using the phospho-antibodies as an indicator of kinase activation. Sensitivity to Silmitasertib was measured using the BrdU Cell Proliferation Assay.

\section{Results:}

FER, PAK4, LIMK1, and PIK3CD were all overexpressed in the TKTB34-RAS and MKTB34RAS cells compared to KTB34-hTERT and TKTB34-PIK3CA cells. The tested concentration range for Silmitasertib $(500 \mathrm{nM}$ to $5 \mu \mathrm{M})$ was ineffective in killing the RAS-transformed cells and was overly toxic to "normal" cells.

\section{Conclusion and Potential Impact:}

FER, PAK4, LIMK1, PIK3CD, and CK2 are potential therapeutic targets for breast cancer metastasis. However, Silmitasertib may not be a good candidate as it is more toxic to "normal" cells compared to cancer cells. The isogenic "normal" and transformed cell line model system described here may help to discover new targets and drugs that kill cancer but not normal cells. 